\title{
Waxing and Waning Presentation of Isolated Cardiac Sarcoidosis on Sequential ${ }^{18}$ F-FDG PET Examinations
}

\author{
Wesam Ostwani, Carolyn Hanna, Aaron E. Brice, and David C. Wymer \\ Department of Cardiac Imaging, Malcom Randall VA Medical Center, College of Medicine, University of Florida, Gainesville, \\ Florida
}

We describe the case of a patient who had suspected myocardial ischemia, showed normal findings on multiple perfusion scans, and showed isolated cardiac sarcoidosis on ${ }^{18} \mathrm{~F}-\mathrm{FDG}-$ PET. Also discussed are the diagnosis and the monitoring of disease response using imaging follow-up.

Key Words: isolated cardiac sarcoidosis; cardiac sarcoidosis; ${ }^{18}$ F-FDG PET

J Nucl Med Technol 2020; 48:378-380

DOI: $10.2967 /$ jnmt.120.246322 was performed. The initial ${ }^{18} \mathrm{~F}-\mathrm{FDG}$ PET study demonstrated lateral left ventricular wall uptake (Figs. 2, 3A, and 4A) suggestive of cardiac sarcoidosis. No extracardiac sarcoidosis was found on workup. Left ventricular endomyocardial biopsy was not performed because it was likely to have a low yield and present an elevated risk. Contrast-enhanced MRI was also contraindicated because of the patient's end-stage renal disease. The patient began taking $40 \mathrm{mg}$ of prednisone daily for 5 mo, and his pacemaker was upgraded to a defibrillator. After 3 mo of treatment, a repeat ${ }^{18} \mathrm{~F}-\mathrm{FDG}$ PET examination showed a partial response, with a significant reduction in

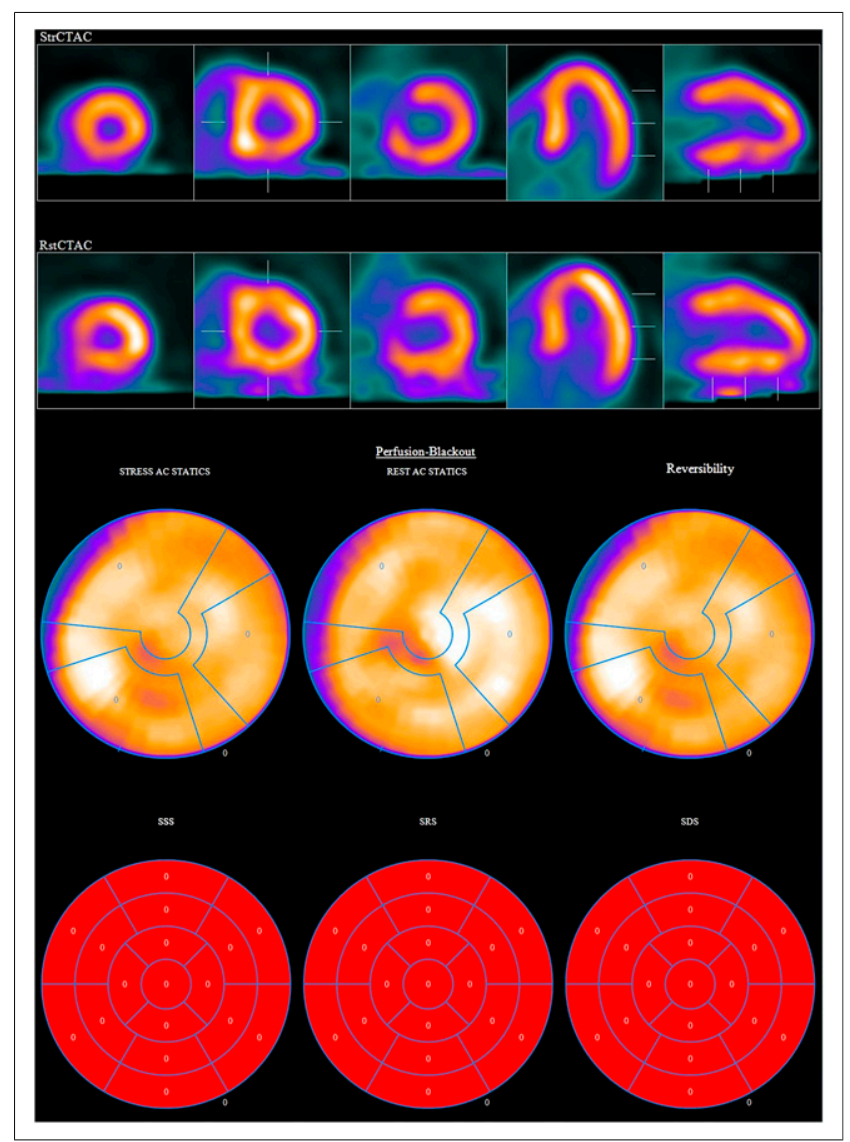

FIGURE 1. Myocardial perfusion scan with normal findings. $A C=$ attenuation-corrected; SDS = summed difference score; SRS = summed rest score; SSS $=$ summed stress score. 


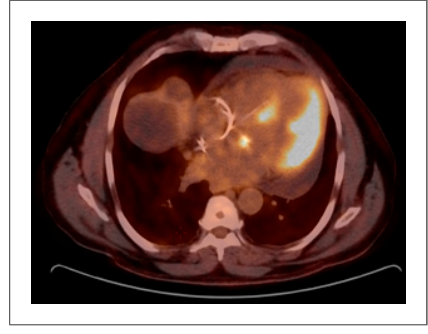

FIGURE 2. Axial ${ }^{18} \mathrm{~F}-\mathrm{FDG}$ $\mathrm{PET} / \mathrm{CT}$ image showing intense uptake in left ventricular lateral wall and lesser septal uptake.

uptake in the lateral left ventricular wall but new uptake in the septal wall (Figs. 3B and 4B).

The patient began taking mycophenolate mofetil, but this was stopped after 1 mo because of oral sores related to herpes virus. His prednisone dose was decreased to $20 \mathrm{mg}$ per day. Repeat ${ }^{18} \mathrm{~F}$-FDG PET after 3 mo revealed a continued response to treatment in the lateral wall but persistent uptake in the septal wall and new uptake in the basal-to-mid-anterior wall (Figs. 3C and 4C).

Currently, the patient is stable, with no cardiovascular symptoms or evidence of worsening cardiomyopathy or ventricular tachycardia. He has continued taking $20 \mathrm{mg}$ of prednisone per day, with plans to restart mycophenolate at a lower dose and titrate upward as tolerated.

\section{DISCUSSION}

On the basis of available data, the approximate rate of isolated cardiac sarcoidosis among patients with cardiac sarcoidosis is $25 \%$. There is limited sensitivity for the diagnosis of cardiac sarcoidosis, with current criteria requiring evidence of extracardiac disease or positive results on endomyocardial biopsy, which itself has limited sensitivity $(20 \%-40 \%)$ because of the patchy nature of the disease. Diagnostic sensitivity for isolated cardiac sarcoidosis is therefore even more limited. Advanced cardiac imaging

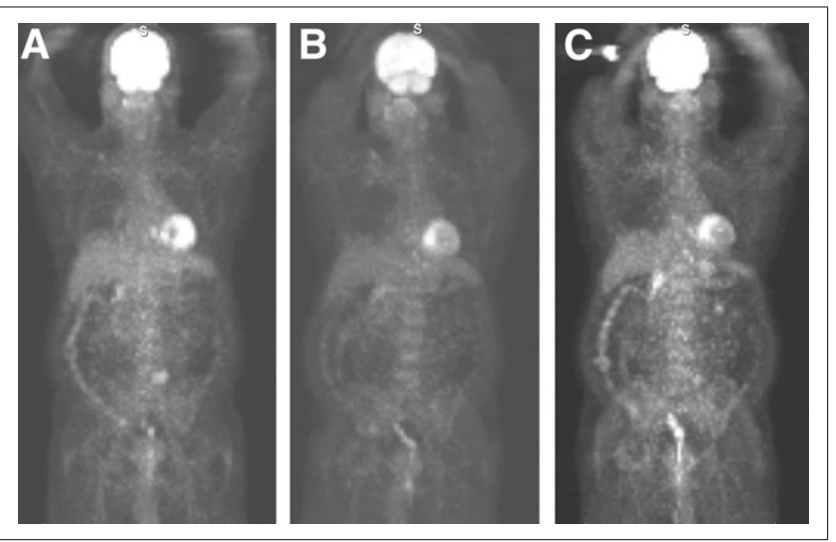

FIGURE 3. Whole-body ${ }^{18}$ F-FDG PET images obtained at 3-mo intervals, showing no extracardiac uptake. (A) Initial examination, with intense lateral wall uptake. (B) Improved findings in lateral wall but new basal-to-mid-septal uptake while patients was taking $40 \mathrm{mg}$ of prednisone daily. (C) Persistent uptake in septal wall and new uptake in basal-to-mid-anterior wall while patient was taking $20 \mathrm{mg}$ of prednisone daily.
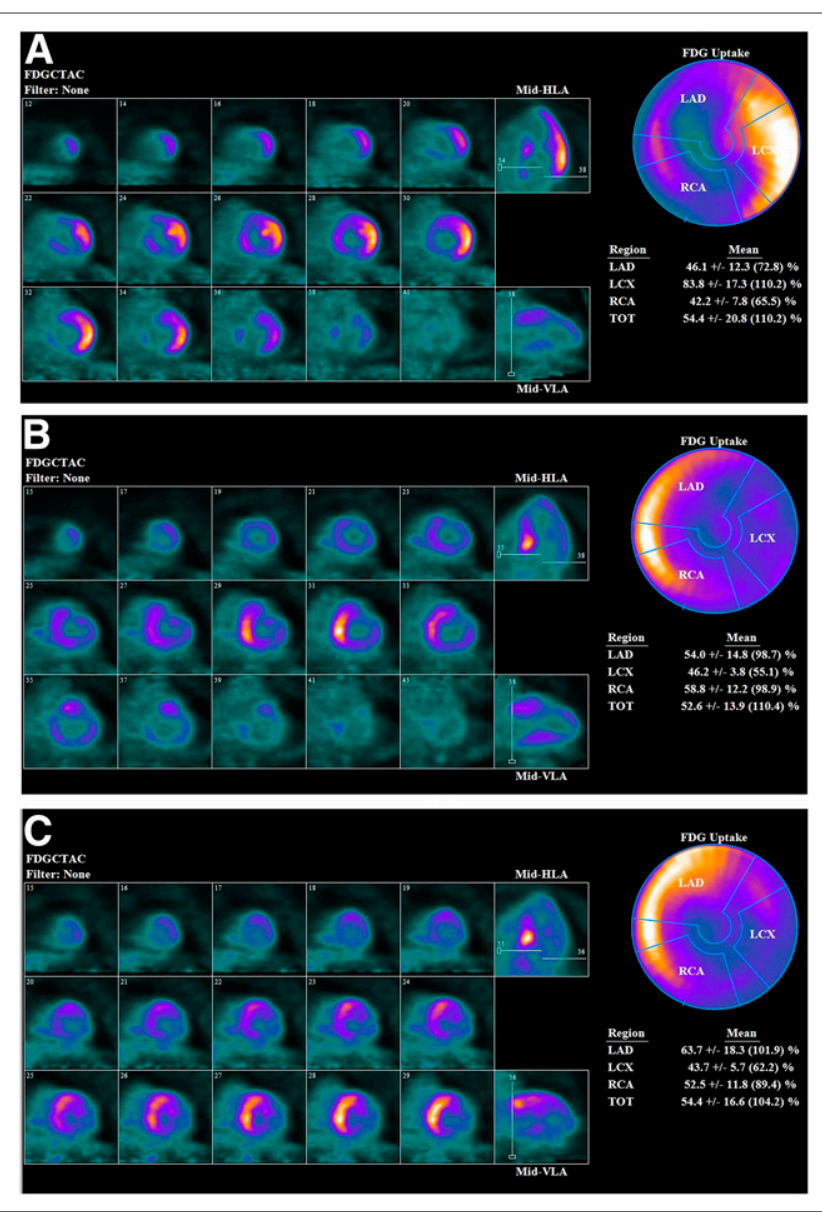

FIGURE 4. Cardiac ${ }^{18}$ F-FDG PET metabolism at 3-mo intervals, including axial, horizontal long-axis, vertical long-axis, and bull'seye views that again show uptake pattern seen in Figure 3. FDGCTAC = CT attenuation-corrected ${ }^{18} \mathrm{~F}-\mathrm{FDG}$ images; HLA = horizontal long axis; $L A D=$ left anterior descending coronary artery; LCX = left circumflex coronary artery; RCA = right coronary artery; TOT $=$ total; VLA $=$ vertical long axis.

techniques using a combined approach of ${ }^{18} \mathrm{~F}$-FDG PET and cardiac MRI could improve early detection of isolated cardiac sarcoidosis and improve the accuracy of diagnosis by providing data on both inflammation and scarring or fibrosis. Whole-body ${ }^{18} \mathrm{~F}-\mathrm{FDG}$ PET is also useful for screening for extracardiac involvement (2). Cardiac MRI was not performed for our patient because of his history of kidney failure and the risk for nephrogenic systemic fibrosis. Endomyocardial biopsy was not performed because of its low sensitivity, pacemaker dependence, and increased risk.

Most experts agree that corticosteroids with or without immunosuppressive therapy should be used in the treatment of cardiac sarcoidosis. ${ }^{18} \mathrm{~F}$-FDG PET reassessment of response to treatment is recommended at 3-mo intervals (1). Our patient showed a mixed response to treatment at both the 3-mo and the 6-mo intervals (Figs. 3 and 4). The data are insufficient to guide management for patients with a mixed response. 


\section{CONCLUSION}

Diagnosis of isolated cardiac sarcoidosis is currently not well defined, and symptoms can overlap those of myocardial ischemia. The presence of ventricular tachycardia and a high-grade atrioventricular block should raise suspicion that cardiac sarcoidosis may be present. Given the variable response to current therapy, frequent 3-mo follow-up with cardiac imaging and monitoring of clinical response is recommended to guide treatment.

\section{DISCLOSURE}

No potential conflict of interest relevant to this article was reported.

\section{REFERENCES}

1. Birnie DH, Nery PB, Ha AC, Beanlands RS. Cardiac sarcoidosis. J Am Coll Cardiol. 2016;68:411-421.

2. Okada DR, Bravo PE, Vita T, et al. Isolated cardiac sarcoidosis: a focused review of an under-recognized entity. J Nucl Cardiol. 2018;25:1136-1146. 